\title{
Evaluation of Liver Function tests (AST \& ALT) in Patients with Hepatitis B and C in Tabriz-Iran (2013)
}

\author{
Navid Sarakhs Asbaghi ${ }^{1}$, Kazem Ghahreman Zadeh $^{2}$, Taher Faraj Zadeh ${ }^{1}$, Javid Lotfi Attari ${ }^{1}$, Zahra Javan \\ Masoomi $^{1}$, Rana Faraj Zadeh ${ }^{1}$, Mohammad Reza Tarmohammadi ${ }^{1}$, Alireza Bakhtarai ${ }^{1}$, Behzad Bahram Zadeh ${ }^{1}$ \\ and Babak Morshed Zadeh ${ }^{1}$ \\ 1. Faculty of Basic Sciences, Department of Genetics, Islamic Azad University, Tabriz 51589, Iran \\ 2. Clinical Central Lab of Province, Tabriz 51589, Iran
}

\begin{abstract}
Viral hepatitis is among the infections that primarily affect the liver and is one of the main causes of death in the world. Every year, more than one million people worldwide die of viral hepatitis. In recent decades, the number of people with hepatitis B and C has declined in Iran. The purpose of this study was to investigate normal and abnormal liver enzymes (AST, ALT) in patients with chronic hepatitis B and C in a number of public and private laboratories in Tabriz. In the study conducted in 2013, of those who had referred to clinical laboratories for various reasons or who had been reported by centers of infectious or dialysis therapy, a sample of 1,000 patients were identified with hepatitis B and C; 693 people had hepatitis B and 307 people had hepatitis C. On a sample of patients, liver enzymes were evaluated using standard methods. The percentage of women and men in this study were inconsistent with global statistics. However this inconsistency could be justified by the alcohol consumption and an increase in the number of addicted people in society as well as women's fear due to some social issues.
\end{abstract}

Key words: Hepatitis B \& C, liver function tests, AST \& ALT, Tabriz.

\section{Introduction}

Hepatitis is the inflammation of liver and is believed to disrupt the activity of it. Hepatitis B virus (HBV) belongs to the family of Hepadnaviridae. It only attends limited number of hosts. It sometimes infects pancreas and kidneys of humans and monkeys.

This small capsidated virus has a double-stranded, circular DNA with a single-stranded part and contains RTase (reverse transcriptase enzyme) which is attached to the virus genome and represents ribonuclease activity. Hepatitis B infection causes liver problems, kidney problems, liver cirrhosi, and high risk of liver cancer. However, another risk that threatens people with chronic hepatitis $B$, is simultaneous infection to hepatitis D [1]. Hepatitis C virus belongs to the Flaviviridae family. It is a small virus coated with positive single-stranded RNA and

Corresponding Author: Navid Sarakhs Asbaghi, M.Sc., research field: genetic and clinical laboratory medicine. E-mail: dr_navid_asbagi@yahoo.com. infection with this type of hepatitis causes liver dysfunction, and liver failure resulting in the liver cirrhosis and liver cancer in rare cases [1, 2].

Hepatitis B infection is a major health concern of the world and a leading cause of liver cancer. About 400 million people around the world are infected with this disease, from which $75 \%$ are Asian. Based on an estimation by World Health Organization, each year about 1.2 million people affected by the hepatitis $B$ virus lose their lives $[3,4]$. Among these people, only $10 \%$ are diagnosed with a chronic illness. The prevalence of $\mathrm{HBV}$ infection in different parts of the world can be divided into three categories: low prevalence (less than 2\%) in some parts of America, Australia and northern Europe; medium prevalence (2\%-7\%) as in many parts of Asia, North Africa and eastern regions of South America and high prevalence (over 8\%) as in Africa, coasts of South East Asia and Alaska. In areas with high prevalence, infection usually occurs at birth. In other parts of the world, 
infection usually occurs after puberty during sexual contact or contact with the infected blood or infected secretions [5-7]. But there are vaccines to protect people against this disease and by getting a full course of injection of the vaccine, people can almost be protected against the virus; yet medical treatment of the infection is expensive and almost ineffective. In contrast to type $\mathrm{B}$, hepatitis $\mathrm{C}$ is asymptomatic and may only cause nonspecific symptoms such as colds and anorexia [ 8, 9].

Differences of acute and chronic hepatitis B are as follows:

Hepatitis B infection can be short-term (acute) or long term (chronic).

Acute hepatitis B infection lasts less than six months. If the disease is acute, the immune system can usually remove the virus from the body. Many adults with hepatitis B are suffering the acute one.

Chronic hepatitis B takes six months or more. When the immune system cannot fight the virus, hepatitis B becomes life-long and can cause serious diseases such as cirrhosis or liver cancer. Most infants and children aged 1 to 5 years are usually infected with chronic hepatitis B. Infection to the disease may not be identified for a long time until it causes an extreme liver disease. The average incubation period of disease is about 90 days (60 -150 days) [10].

Transmission of hepatitis viruses B and C occurs through:

(1) Sexual contact (the main route of transmission): unsafe sex, a transfer from an infected partner, multiple and unknown sexual partners;

(2) Transfer by means of sharp equipment, and under skin contacts: sharing the same drug injection equipment by addicted people (one of the most common routes of transmission of hepatitis C), tattooing, acupuncture, ear piercing, injections, circumcision, cupping and unsafe medical and dental practices;

(3) Blood transfusions, organ transplants, and blood products: through contaminated blood transfusion that mostly happened in the past. Fortunately, with the introduction of blood safety policies, contamination in this way has been dramatically prevented;

(4) Mother-to-child transmission (vertical transmission), especially in the last trimester of pregnancy. If the mother is infected, the probability of mother-to-child transmission is high, and it has been reported to be one of most common routes of transmission of hepatitis $\mathrm{B}$ in Iran. On the transmission of infection through breast feeding, there is much debate. Although HBsAg is isolated from breast milk, due to a very close contact between the baby and mother, the transmission cannot surely be attributed to the breast milk;

(5) Transfer during diagnostic and therapeutic services: transmission through contaminated instruments in dentistry, dialysis, endoscopy, surgical and diagnostic procedures and treatments, needle stick injuries, particularly for medical staff;

(6) Body fluids: such as bile, feces, semen, and vaginal discharge that are considered a route for virus transmission;

(7) Other: use of common items such as tooth brush, blade, baby bottle, knife, scissors and so on [11-14].

The virus is asymptomatic in patients; they carry it chronically and for a long time in their blood, and transmit it to healthy individuals by the mentioned ways. In an investigation, it was proved that people with HIV are more susceptible to particular types of HBV and HCV. Coming up with $\mathrm{HIV}+$ and hepatitis infection at the same time, causes rapid progress in liver problems and further damage to the liver in a short time and thus it is more life-threatening [7].

According to the common transmission ways of both viruses, the possibility exists that the person is suffering from simultaneous infection to hepatitis $B$ and $\mathrm{C}$; yet there may be a number of people who are also infected with HIV. This is mostly seen among intravenous drug users.

Symptoms of hepatitis B: 
Signs and symptoms of hepatitis B usually appears after three months of infection and can include a wide range of symptoms. such as [1]:

- Abdominal pain

- Dark urine

- Fever

- Aching joints and muscles

- Decrease or loss of appetite

- Nausea and vomiting

- Fatigue and weakness

- Yellowing of the skin and the eyes appearing whiter

- Rash or skin rash

Symptoms of hepatitis C:

Hepatitis $\mathrm{C}$ generally does not show any symptoms, but if there are any, they can include [1]:

- Fatigue

- Fever

- Aching joints and muscles

- Nausea and poor appetite

- Yellowing of the skin and the eyes appearing whiter

- Yellow or pale stools

Investigated tests (AST \& ALT) were used for patients with hepatitis at the request of physicians to diagnose liver injuries, and help identify liver diseases and suspected cases of liver disorders such as hepatitis. AST enzyme is produced in all cells throughout the body, but there is much volume of it in the heart and liver, and to a lesser extent in the kidneys and muscles. In healthy individuals, the level of AST is low in the blood. When the liver cells or muscles are damaged, AST is released into the blood. Thus, AST test is a useful test for the diagnosis of liver damage. The ALT enzyme can also be requested in cases of suspected liver disease and liver damage. This enzyme is also present in the cells of liver and kidneys, and smaller amounts of it can be found in the heart and muscles. In healthy individuals, the level of ALT is low in the blood. When the liver is damaged, usually before more obvious symptoms of liver injury, such as jaundice, ALT enters the blood stream. This makes ALT a useful test for the diagnosis of liver damage. These two tests are often asked as part of a liver panel for the screening and diagnosis of liver disorders, although ALT is more specific than AST. Level of AST and ALT is not too high in patients with chronic hepatitis, often lower than four times of the normal level. In chronic hepatitis, level of these enzymes is varied between normal and less increase. In most liver diseases, the ALT level is higher than AST's and as a result, the ratio of ALT \& AST would be low. There are a few exceptions; The ratio of ALT/AST is usually high in alcoholic hepatitis, cirrhosis, and muscle damage. Therefore, if the ratio of ALT/AST is less than one, it indicates mild liver damage, and if its value is greater than one, it indicates that the damage is severe or it is a chronic liver disease $[13,15]$.

\section{Materials and Methods}

In this study which was carried out in 2013, of those who had referred to clinical laboratories for various reasons or who had been reported by centers of infectious or dialysis therapy, a sample of 1000 patients were identified with hepatitis B and C; 693 people had hepatitis B and 307 people had hepatitis C. Blood samples were obtained from patients and sampling required no specific preparation. Patients with hepatitis were diagnosed was using the serological methods of ELISA, RIBA, ECL, Western blot and PCR. Liver enzymes of aspartate aminotransferase (AST; SGOT) and alanine aminotransferase (ALT; SGPT) were evaluated in the blood samples using standard method of enzymatic photometry and also with the help of autoanalyzer device.

\section{Results and Discussion}

Getting vaccines and following health instructions are the best ways to avoid hepatitis B. Hepatitis B vaccine which is currently used is actually the same antigen of hepatitis B virus (HBsAg) which is 
obtained from the blood of healthy carriers and condensed. There is another type of vaccine that has been prepared using genetic methods (recombinant vaccines). Vaccination is usually done three times in intervals of one month and six-month from the first injection. Vaccination represents few side effects such as redness and swelling at the injection site, sometimes mild muscle aches, and fever which is less than $38^{\circ} \mathrm{C}$ and disappears after a while [1]. In addition to vaccination, other ways to prevent and reduce the risk of hepatitis B include: (1) avoidance of risky behaviors and unprotected sex; (2) quitting taking narcotics and illegal drugs; (3) using a new condom for each sex; (4) considering hygiene in tattooing; (5) not using shared instruments such as needles, syringes, toothbrushes and razors. However in the case of hepatitis C, it must be stated that unfortunately there is not any vaccine to prevent hepatitis $\mathrm{C}$ yet. The available vaccines are for hepatitis A and B. However, there are ways to prevent the disease, like those abovementioned for hepatitis B [1].

Patients' demographic data and statistics, and the results of analysis of patients' statistics along with their liver tests are as follows:

According to the statistics mentioned in this paper, some suggestions for prevention and reduction of patients with these infections are expressed, given that hepatitis is one of the major problems in health field in the world, and causes and ways of transmission of hepatitis are different depending on the health status, traditions and social customs, and moral issues. In Iran, the majority of patients with liver disease are not identified due to the absence of symptoms and in most cases, the infected person is identified after disease development. Of course, it should be noted that increasing awareness should not spread misplaced fear

Table 1 Patients' demographic data and statistics of patients and liver tests.

\begin{tabular}{ll}
\hline The age range of patients & A month-88 Year \\
\hline The mean age of the patients & $37 \pm 6$ \\
Number of males & $58 \%$ \\
Number of females & $42 \%$ \\
The number of people with hepatitis B among 1000 Person & 693 people \\
The number of people with hepatitis C among 1000 Person & 307 people \\
Percentage of patients with abnormal liver tests & $28 \%-0 \%$ \\
Percentage of patients with both diseases simultaneously & $2 \%-3 \%$ \\
Percentage of people with hepatitis B in Tabriz & $\sim 0.3 \%$ \\
Percentage of people with hepatitis C in Tabriz & $\sim 0.14 \%$ \\
\hline
\end{tabular}

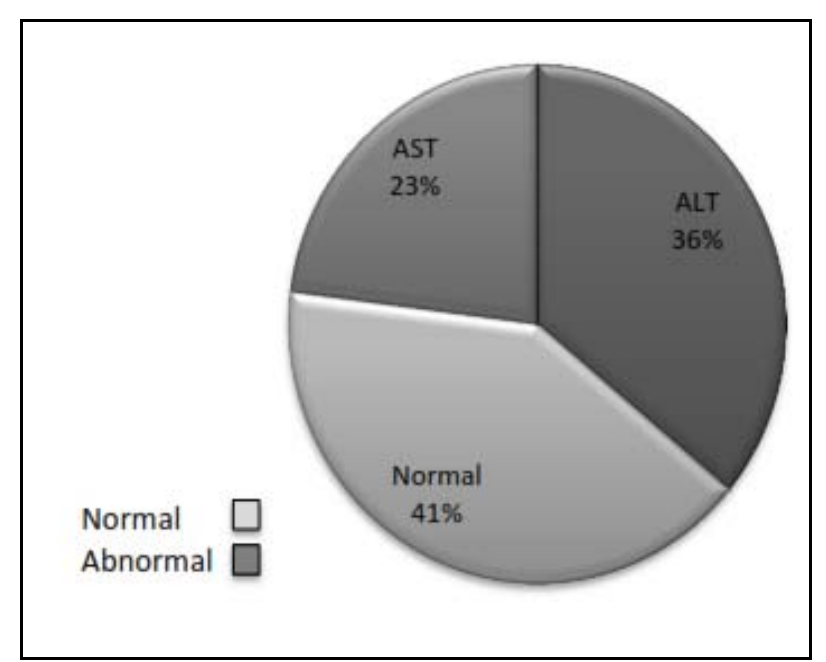

Fig. 1 Rate of Abnormal Liver Tests in Patients with Hepatitis B.

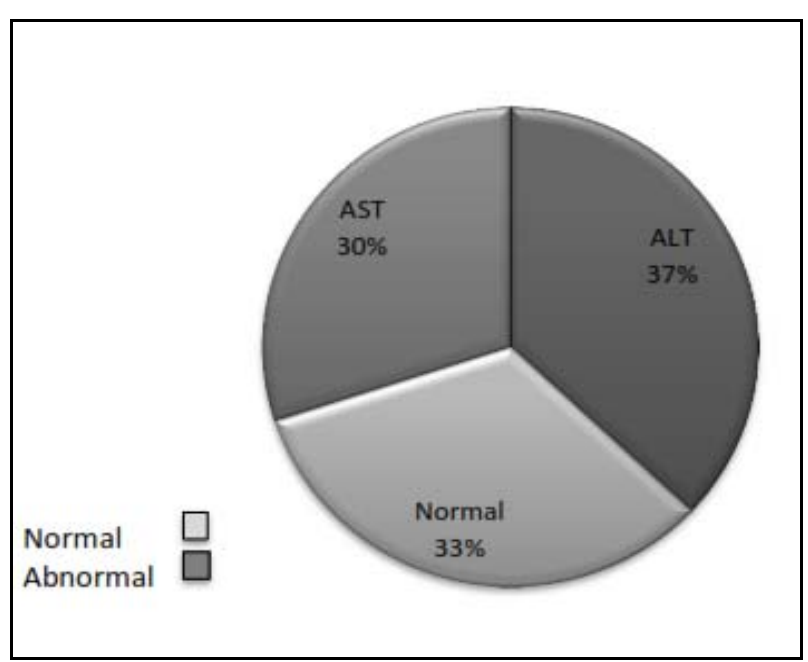

Fig. 2 Rate of Abnormal Liver Tests in Patients with Hepatitis C. 


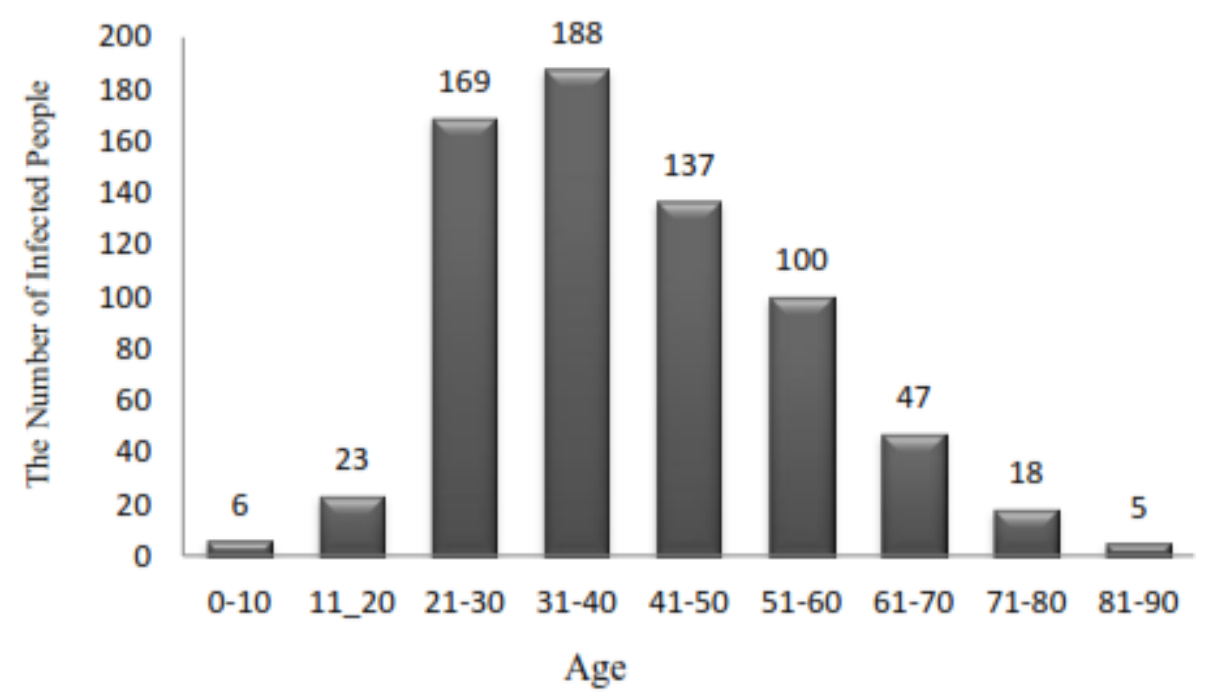

Fig. 3 The Age Distribution of Those Affected With Hepatitis B Referring Public And Private Laboratories in Tabriz in 2013.

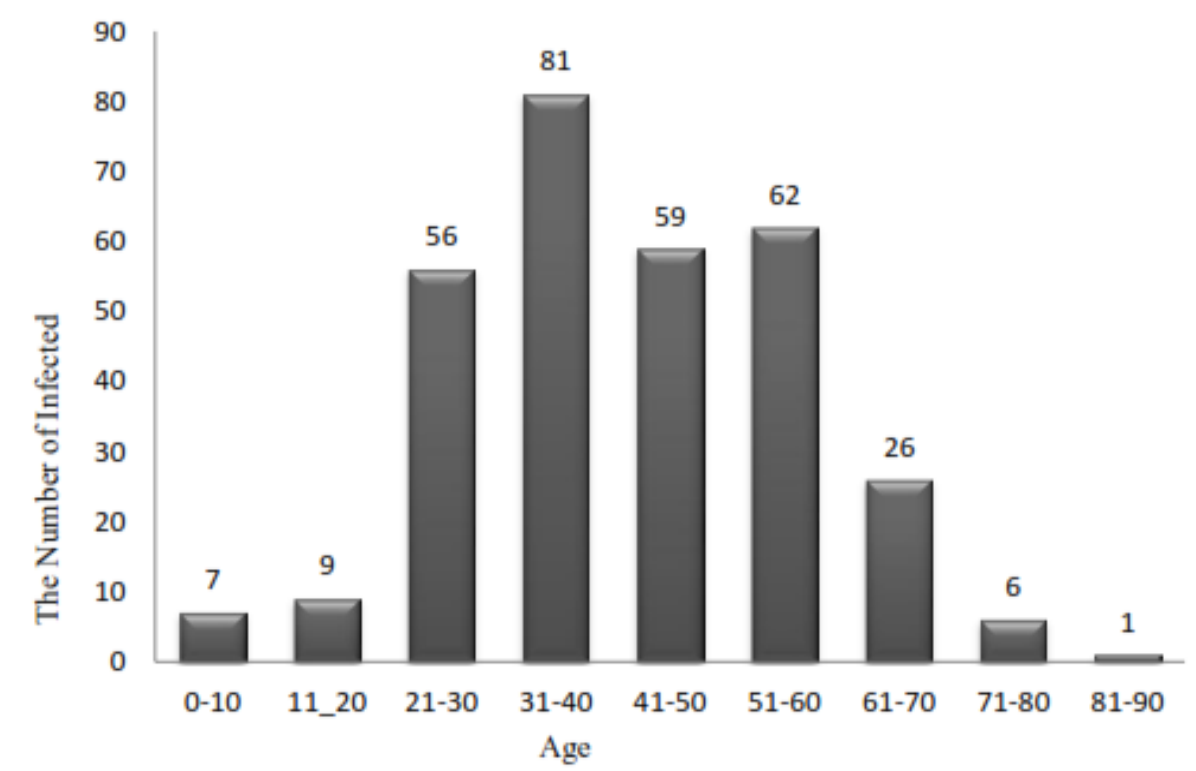

Fig. 4 The Age Distribution of Those Affected With Hepatitis C Referring Public And Private Laboratories in Tabriz in 2013.

in society. Obviously, infected patients should be supported materially and spiritually and there should be cooperation to eradicate the disease. In Iran, hepatitis $\mathrm{B}$ is one of the major health problems and about 2-7 percents of the population suffer from this disease in some forms. According to the latest statistics, about 2,000,000 people in the country are infected with HBV and about 200,000 people with hepatitis C. The risk of getting hepatitis B during lifetime is about $20-60 \%$, and the infection can occur at all ages [14, 15]. Hence in Iran, due to high infections, critical actions should be done to raise public awareness, promote social and personal hygiene, and educate high risk groups. It is recommended that all individuals at risk (physicians and paramedics, injecting drug users, dialysis patients, and recipients of blood) and newborn babies get hepatitis B vaccine, as vaccination is the most effective and inexpensive means to prevent hepatitis $\mathrm{B}$. As mentioned, the prevalence of hepatitis $\mathrm{C}$ is low 
compared to Hepatitis B, and since most people with hepatitis $\mathrm{C}$ seem to have chronic hepatitis, problems related to hepatitis $\mathrm{C}$ increase. For men, the diagnosis test of hepatitis is recommended at the time of admission to military service, employment, and also marriage, and women should be screened in employment, marriage, and pregnancy to prevent the increase of number of patients. For those addicted people, identifying them and giving them disposable infusion devices could prevent the prevalence of infections [15]. In order to support the affected individuals financially and spiritually, health and insurance officials should help for the diagnosis and treatment through affording the medical costs. These patients have the right to live in society like other people who have intellectual property rights, so they should not be wrongly judged. Then we should not spread unnecessary fear in society, and efforts should be made to vaccinate people as it is the most effective and cost-effective means of preventing hepatitis B. Following health instructions and safety issues is also the way to prevent hepatitis C.

\section{Acknowledgments}

We sincerely appreciate who helped us through this work in Pardis Specialized and Ultra Specialized Lab. (Mrs. Nasiri and Mrs. Feizi), Danesh Laboratory (Mr. Afsari), Laboratory of Imam Reza Hospital (Mr. Masoud far), Laboratory of Sina Hospital (Mr. Khosroshahi), Laboratory of Nor-e-nejat Hospital (Mr. Barezman) and Central State Laboratory (Dr. Norouzi, Mr. Abbasi and Mr. Radmehr).

\section{References}

[1] Alavian, S. M., Adibi, P., and Zali, M. R. 2005. "Heptitis C Virus in Iran: Epidemiology of an Emerging Infection.” Arch. Iranian Med. 8:84-90

[2] Rosen, H. R. 2011. "Clinical practice. Chronic hepatitis C infection.” The New England journal of medicine 364
(25): 2429-38

[3] Lee, W. M. 1997. “Hepatitis B Virus Infection.” N. Engl. J. Med. 337: 1733-45.

[4] Alavian, M. 2006. .”Immunization: An Important Strategy to Control Hepatitis B.” Hepatitis Monthly 6 (1) 3-5.

[5] Lavanchy, D. 2004. "Hepatitis B Virus Epidemiology, Disease Burden, Treatment, and Current and Emerging Prevention and Control Measures.” J. Viral. Hepat. 11: 97-107.

[6] Kowdley, K. V. 2004. "The Cost of Managing Chronic Hepatitis B Infection: A Global Perspective.” J. Clin. Gastroenterol. 38: S132-S133

[7] Mast, E. E., Weinbaum, C. M., Fiore, A. E., Alter, M. J., Bell, B. P., Finelli, L., Rodewald, L. E., Douglas, J. M. Jr., Janssen, R. S., Ward. J. W., 2006. “A Comprehensive Immunization Strategy to Eliminate Transmission of Hepatitis B Virus Infection in the United States." http://www.cdc.gov/mmwr/preview/mmwrhtml/rr5516a1. htm?s_cid=rr5516a1_e.

[8] WHO. 2002. Hepatitis B. WHO. WHO/ CDS/ CSR/ LYO/ 2002.2.

[9] McMahon, B. J., Helminiak, C., Wainwright, R. B., Bulkow, L., Trimble, B. A., and Wainwright, K. 1992. "Frequency of Adverse Reactions to Hepatitis B Vaccine in 43618 persons.” Am. J. Med. 92 (3): 254-6.

[10] Krugman, S., Overby, L. R., Mushahwar, I. K., Ling, C. M., Frosner, G. G., and Deinhardt, F. 1979. "Viral Hepatitis, type B. Studies on Natural History and PreventionRe-examined.” N. Engl. J. Med. 300: 101-6.

[11] Alavian, S. M, Einollahi, B., Hajarizadeh, B., Bakhtiari, S., Nafar, N., and Ahrabi S. 2003. "Prevalence of Hepatitis C Virus Infection and Related Risk Factors among Iranian Haemodialysispatients.” Nephrology 8: 256-60.

[12] WHO. Hepatitis B immunization. WHO. 2001; WHO/ $\mathrm{V} \& \mathrm{~B} / 01.28$.

[13] Assy, N., and Minuk, G. Y. 1995. "Serum Aspartate Aminotransferase (AST) Values as a Useful Predictor for the Histologic Features of Chronic Hepatitis C Infection in Adults.” Hepatology 22: 272. (Abstract)

[14] WHO 2001. Introduction of Hepatitis B Vaccine into Childhood Immunization Services. WHO. WHO/V\&B/01.31.

[15] Saberifiroozi, M. 2009. "Development of Gastroenterology and Hepatology in Iran: Part II-Advances in Research and Therapeutic Modalities.” Arch. Iranian Med.12 (5): 516-25. 